\title{
Conhecimento dos estudantes de enfermagem do centro universitário Estácio de Sergipe sobre a população LGBTQIAP+
}

\author{
Nursing students' knowledge of the Estácio University Center of Sergipe about the \\ LGBTQIAP + population

\section{Conocimiento de los estudiantes de enfermería del Centro Universitario Estácio de Sergipe sobre la población LGBTQIAP +}

Tereza Monique Côrtes Gomes ${ }^{1 *}$, Carlos Mágno Santos Barbosa ${ }^{1}$, Andriellen Rabelo Carvalho ${ }^{1}$, André Luiz de Jesus Morais ${ }^{1}$, Taciana Silveira Passos², Ana Fatima Souza Melo de Andrade'.

\section{RESUMO}

Objetivo: Identificar a percepção dos acadêmicos de enfermagem acerca dos paradigmas da diversidade sexual. Métodos: Trata-se de estudo descritivo exploratório com abordagem quantitativa, realizada no Centro Universitário Estácio de Sergipe com 53 acadêmicos do curso de graduação de enfermagem, do município de Aracaju/SE, no período de agosto a setembro de 2020. Resultados: Evidenciou-se maior proporção de discentes que se sentiam capacitados em realizar atendimento entre aqueles que conheciam plenamente o significado da sigla LGBTQIAP+ $(p=0,004)$ e aqueles que estavam cientes da existência de alguma política de amparo à saúde para essa população $(\mathrm{p}=0,042)$. Existiu distribuição significativa entre ter tido contato com a temática em alguma disciplina e conhecer a sigla $(p=0,004)$, saber diferenciar sexo biológico, identidade de gênero, expressão de gênero e orientação sexual $(p=0,015)$, estar ciente da existência de alguma política de amparo à saúde para essa população $(p=0,005)$, e percepção de peculiaridades do público LGBTQIAP+ que necessitam de maior atenção no âmbito da saúde $(p=0,030)$. Conclusão: Conclui-se que o nível de conhecimento dos estudantes acerca das singularidades e políticas de saúde direcionadas à população LGBTQIA+ é determinante para oferta de uma assistência integral que reconheça os contextos de vulnerabilidade e exclusão a que essa população está exposta.

Palavras-chave: Diversidade de gênero, Educação em enfermagem, Estudantes, Saúde.

\begin{abstract}
Objective: To identify the perception of nursing students about the paradigms of sexual diversity. Methods: This is an exploratory descriptive study with a quantitative approach, carried out at the Estácio de Sergipe University Center with 53 undergraduate nursing students from the municipality of Aracaju / SE, from August to September 2020. Results: A greater proportion of students who felt able to provide care among those who fully knew the meaning of the acronym LGBTQIAP + $(p=0.004)$ and those who were aware of the existence of some health support policy for this population ( $p$ $=0.042$ ). There was a significant distribution between having had contact with the theme in some discipline and knowing the acronym $(p=0.004)$, knowing how to differentiate biological sex, gender identity, gender expression and sexual orientation ( $p=0.015)$, being aware of the existence of some health support policy for this population $(p=0.005)$, and perception of peculiarities of the LGBTQIAP + public that need more attention in the area of health $(p=0.030)$. Conclusion: It is concluded that the level of knowledge of students about the singularities and health policies directed to the LGBTQIA + population is a determining factor in offering comprehensive care that recognizes the contexts of vulnerability and exclusion to which this population is exposed.
\end{abstract}

Key words: Gender diversity, Nursing education, Students, Health.

\section{RESUMEN}

Objetivo: Identificar la percepción de los estudiantes de enfermería sobre los paradigmas de la diversidad sexual. Métodos: Se trata de un estudio descriptivo exploratorio con enfoque cuantitativo, realizado en el Centro Universitario Estácio de Sergipe con 53 estudiantes de pregrado en Enfermería del municipio de Aracaju / SE, de agosto a septiembre de 2020. Resultados: Una mayor proporción de estudiantes que se sintieron capaces de brindar atención entre los que conocían plenamente el significado de la sigla LGBTQIAP + $(p=0,004)$ y los que conocían la existencia de alguna política de apoyo a la salud para esta población $(p=0,042)$. Hubo una distribución significativa entre haber tenido contacto con el tema en alguna disciplina y conocer la sigla $(p=0,004)$, saber diferenciar sexo biológico, identidad de género, expresión de género y orientación sexual $(p=0,015)$, ser consciente de la existencia de alguna política de apoyo a la salud para esta población $(p=0,005)$, y percepción de las peculiaridades del público LGBTQIAP + que requieren mayor atención en el área de salud $(\mathrm{p}=0,030)$. Conclusión: Se concluye que el nivel de conocimiento de los estudiantes sobre las singularidades y políticas de salud dirigidas a la población LGBTQIAP + es un factor determinante para ofrecer una atención integral que reconozca los contextos de vulnerabilidad y exclusión a los que está expuesta esta población.

Palabras clave: Diversidad de género, Educación en enfermeira, Estudiantes, Salud.

${ }^{1}$ Centro Universitário de Sergipe, Aracaju - SE. *E-mail: nickcortess@gmail.com

2 Universidade Tiradentes, Aracaju - SE. 


\section{INTRODUÇÃO}

O conhecimento sobre a população Lésbicas, Gays, Bissexuais, Transexuais ou Transgênero, Queer, Intersexo, Assexual, Panssexuais e mais que acrescenta outras orientações e identidade de gênero (LGBTQIAP+) ainda é muito limitado, embora esse grupo, considerado uma minoria, tenha obtido alguns direitos ao longo do tempo. Atrelado a essa comunidade, a identidade de gênero e orientação sexual são assuntos de grande importância para a compreensão da sigla, que por sua vez sempre está em constante modificação. Desse modo, a identidade de gênero é a maneira que o indivíduo tem de se perceber aos gêneros, que pode ser ao masculino ou feminino, ou às outras identidades não binárias (SANTOS JS, et al., 2019).

A orientação sexual caracteriza-se pela atração sexual, afetiva e emocional por diversos, iguais ou diferentes gêneros. Vale ainda observar que para entender a população LGBTQIAP+ ainda no acolher as variedades de expressões de gêneros, que a partir do desenvolvimento social, a referida comunidade vai acrescentando novos conceitos como as populações cross-dressing e queen e da população intersexual (GUIMARÕES NP, et al., 2020).

Dessa forma, fez-se necessário o desenvolvimento de estudos e conhecimentos sobre esse movimento social para que o preconceito e discriminação a esse público sejam extintos da sociedade. $O$ estudo realizado por Negrito FRN, et al. (2019), na Itália, na cidade de Nápoles, com indivíduos do grupo LGBTQIAP+, constatou que $28 \%$ destes sofrem no mínimo algum tipo de violência por parte de outras pessoas em sua vivência, havendo o predomínio de violência verbal, com uma totalidade de $85,2 \%$ dos casos. Já $52 \%$ relataram estados emocionais de infelicidades e 37\% percepções negativas de si. Logo, esses acontecimentos fortalecem o valor do cuidado focado nas necessidades e as qualidades específicas dos indivíduos LGBTT que sofrem agressão psicológica (ALBUQUERQUE GA, et al., 2016a).

No que se refere à saúde pública, no Brasil, dados apontam que na Parada Gay de São Paulo 14,4\% da população LGBT argumentaram que nos serviços de rede pública de saúde passaram por alguma espécie de discriminação. Por isso, a homofobia (LGBTfobia) é uma das maiores causas de crimes contra homossexuais no país em questão. O Sistema de Informação de Mortalidade do Sistema Único de Saúde (SIM/SUS) aponta que ocorreram 2.511 homicídios dessa categoria no Brasil no período de 1980 a 2005. Dessa forma, a mudança na saúde para o acolhimento à população LGBTQIAP+ resulta também na modificação da maneira do profissional atuar (CARDOSO MR e LUZ NS, 2017). É importante que reconheçam a população LGBTQIAP+ para que o direito à saúde dessa comunidade não seja violado.

No âmbito da saúde pública, as questões ligadas às políticas públicas de saúde desenvolvidas para a assistência integral à população LGBTQIAP+ têm sido objeto de estudos que buscam a efetiva implementação dessas diretrizes, desconstruindo gradativamente a discriminação e o preconceito existentes até os dias atuais e garantindo uma assistência digna e humanizada, respeitando as diferenças (ALMEIDA JSM, et al., 2018).

O problema analisado tem passado por transformações nas últimas décadas com ajuda marcante dos vários movimentos da sociedade civil e áreas governamentais, que vão desde as demandas em relação à epidemia da AIDS até a implementação da Política Nacional De Saúde Integral LGBTQIAP+, alcançando maior visibilidade frente ao SUS na desconstrução dos fatores de vulnerabilidade já vividos historicamente por esse público. Tais mudanças proporcionaram, entre elas, o uso do Direito Social, implantação de cirurgias de resignação de sexo para trans sexuais femininos, visto como um importante e recente avanço para o público. No entanto, nem todos os fatores ainda foram superados (NEGREIROS FRN, et al., 2019).

A formação de profissionais de saúde com um novo olhar no atendimento e prestação dos serviços de saúde faz-se necessária, pois a discriminação e o preconceito são problemas frequentes e perceptíveis. Quando se realiza abordagem aos profissionais de saúde sobre essa temática, obtêm-se respostas, na sua maioria, como desconhecedores das suas atribuições específicas para a prestação de uma assistência em saúde envolvendo a necessidade social (GOMES TMC, et al., 2020).

Diante dessa lacuna, percebeu-se que existe um caminho a ser trilhado com os acadêmicos da área da saúde, ainda no período de formação teórico e prático, levando-os a entender que precisam conhecer 
profundamente as deficiências do trabalho realizado com essa população vulnerável. Nesse sentindo, o estudo proposto é de ampla relevância, uma vez que contribuirá para reflexão sobre fatores que possam interferir futuramente no processo de atendimento em saúde para a população LGBTQIAP+ (FERREIRA BO, et al., 2018).

Dessa forma, possibilitando através dos novos profissionais de saúde a desconstrução de fatores de vulnerabilidade nos serviços de saúde. Por outro viés, possibilitará a elaboração e estruturação de planos de ensino nas instituições acadêmicas dos cursos de enfermagem e de saúde, com uma abordagem eficiente quanto à sexualidade, identidade sexual e atendimento à população LGBTQIAP+. Assim, objetivou-se identificar a percepção dos acadêmicos de enfermagem do Centro Universitário Estácio de Sergipe acerca dos paradigmas da diversidade sexual.

\section{MÉTODOS}

Tratou-se de uma pesquisa de campo do tipo exploratória com caráter quantitativa. Realizada no Centro Universitário Estácio de Sergipe com acadêmicos do curso de graduação de enfermagem, do município de Aracaju, Sergipe, que estão entre o quinto e décimo período, que foi realizada no período de agosto a setembro de 2020. Tendo uma amostra de 53 entrevistados.

Foram incluídos nessa pesquisa acadêmicos do curso de enfermagem, que já cursaram disciplinas práticas de atendimento ao público nas unidades básicas de saúde e em instituições hospitalares (alunos entre o quinto e décimo período da graduação), que aceitaram participar espontaneamente do estudo e assinaram o Termo de Consentimento Livre e Esclarecido, como forma de segurança tanto para o entrevistado quanto para o entrevistador. $O$ critério de exclusão foram os acadêmicos de enfermagem que não cursaram disciplinas práticas de atendimento ao público, estudantes com curso inferior ao quinto período de graduação e que não assinaram o Temo de Consentimento Livre Esclarecido.

Os riscos foram de constrangimento por parte dos entrevistados por não saberem responder as questões que compõem o questionário, que foi minimizado por meio de uma coleta de dados restrita através de um questionário individual de acesso remoto, pelo Google Docs. Os benefícios foram à promoção de capacitações para os acadêmicos do curso de enfermagem e melhoria no perfil do discente de enfermagem do Centro Universitário Estácio de Sergipe.

O instrumento utilizado para a coleta de dados semiestruturado já validado, utilizado nos estudos de Lopes LP, et al. (2019), Zani LF e Terra MF (2019), intitulados por: Diversidades de gêneros e acesso à saúde: concepção dos estudantes de medicina e enfermagem do Centro Universitário de Patos de Minas; e Conhecimentos sobre identidade de gênero e orientação sexual entre graduandos/as de enfermagem, ambos adaptados por meio do formulário Google Docs. Por meio das variáveis dependentes foi identificado o conhecimento dos acadêmicos acerca da temática supracitada; identificado a percepção dos acadêmicos de enfermagem quanto ao papel do enfermeiro no cuidado à população LGBTQIAP+; e identificada as dificuldades dos acadêmicos de enfermagem para a prestação da assistência de saúde à população LGBTQIAP+.

A coletada de dados foi realizada por meio do instrumento de coleta de dados, o questionário estruturado com questões fechadas e aplicado aos acadêmicos de enfermagem do Centro Universitário Estácio de Sergipe com o compartilhamento do link do formulário do Google Docs. Os alunos responderam o instrumento após assinarem o termo de consentimento livre esclarecido.

Esta pesquisa foi submetida para apreciação do Comitê de Ética e Pesquisa (CEP) do Centro Universitário Estácio de Sergipe por meio da na Plataforma Brasil e obedeceu aos critérios de ética em pesquisa com seres Humanos conforme $n^{\circ} 466 / 2012$ do Conselho Nacional de Saúde, CAEE: 36807320.2.0000.8079. Os dados coletados e analisados serão mantidos por cinco anos na posse dos pesquisadores para eventuais comprovações, conforme a Resolução 510/2016 do art. 28, IV. Todos os participantes do estudo foram convidados a autorizar o uso dos dados por meio do Termo de Consentimento Livre e Esclarecido (TCLE), conforme preconiza a Resolução $n^{\circ}$ 466/2012 do Conselho Nacional de Saúde. 
Os dados foram digitados no Excel e processados no Programa Statistical Package for the Social Sciences (SPSS), versão 15.0, e apresentados por meio de estatísticas descritivas. Foi utilizada análise univariada por meio de distribuições de frequências em número absoluto e porcentagem. Posteriormente, o teste Qui-quadrado de Pearson foi utilizado para análise da proporção de distribuição das variáveis. O nível de significância estatística utilizado foi de $5 \%$ ( $p$ menor ou igual 0,05 ).

\section{RESULTADOS}

Participaram da pesquisa 53 estudantes do Centro Universitário Estácio de Sergipe do curso de enfermagem na Tabela 1. Os indivíduos eram predominantemente do sexo feminino (79,25\%), cisgênero (98,11\%), a maioria jovem com 18 a 24 anos $(50,94 \%)$ e solteiro $(69,81 \%)$. A participação com maiores representatividades foram dos discentes do $9^{\circ}$ e $10^{\circ}$ períodos, com uma porcentagem de $(28,35 \%)$ em ambos. Os demais corresponderam a discentes do $8^{\circ}$ período $(20,79 \%)$, do $7^{\circ}$ período $(11,34 \%)$, do $6^{\circ}$ período $(9,45 \%)$ e em menor parte do 5 o período $(1,89 \%)$.

Tabela 1- Caracterização do perfil sócio acadêmico e abordagem à população LGBTQIAP+.

\begin{tabular}{|c|c|c|}
\hline & $\mathbf{N}$ & $\%$ \\
\hline \multicolumn{3}{|l|}{ Sexo atribuído ao nascimento } \\
\hline Feminino & 42 & 79,25 \\
\hline Masculino & 11 & 20,75 \\
\hline \multicolumn{3}{|l|}{ Gênero } \\
\hline Cisgênero & 52 & 98,11 \\
\hline Transgênero/ Transexual & 1 & 1,89 \\
\hline \multicolumn{3}{|l|}{ Faixa etária } \\
\hline 18 a 24 anos & 27 & 50,94 \\
\hline 25 a 45 anos & 25 & 47,17 \\
\hline 46 a 61 anos & 1 & 1,89 \\
\hline \multicolumn{3}{|l|}{ Estado civil } \\
\hline Solteira & 37 & 69,81 \\
\hline Casada & 11 & 20,75 \\
\hline Divorciada & 1 & 1,89 \\
\hline Outros & 4 & 7,55 \\
\hline \multicolumn{3}{|l|}{ Período do curso } \\
\hline 5o período & 1 & 1,89 \\
\hline 6- período & 5 & 9,43 \\
\hline 7º período & 6 & 11,32 \\
\hline 8o período & 11 & 20,76 \\
\hline 9o período & 15 & 28,3 \\
\hline 10 período & 15 & 28,3 \\
\hline \multicolumn{3}{|c|}{$\begin{array}{l}\text { Sente-se capacitado em atender um indivíduo LGBTQIAP+ e suas } \\
\text { particularidades }\end{array}$} \\
\hline Não & 8 & 15,09 \\
\hline Pouco & 13 & 24,53 \\
\hline Moderadamente & 25 & 47,17 \\
\hline Totalmente & 7 & 13,21 \\
\hline \multicolumn{3}{|c|}{$\begin{array}{l}\text { Abordagem em alguma disciplina sobre a melhor conduta frente a } \\
\text { comunidade LGBTQIAP+ }\end{array}$} \\
\hline Não & 19 & 35,84 \\
\hline Pouco & 23 & 43,4 \\
\hline Moderadamente & 10 & 18,87 \\
\hline Totalmente & 1 & 1,89 \\
\hline
\end{tabular}

Fonte: Gomes TMC, et al., 2020.

As variáveis compreendidas pelo sentimento de capacitação no atendimento e no tocante à abordagem frente aos LGBTQIAP+ obteveram os seguintes resultados: $47,17 \%$ responderam que se sentem moderadamente capacitados; $24,53 \%$ pouco capacitados; $15,09 \%$ não capacitados e $13,21 \%$ totalmente capacitados. Em relação à abordagem frente aos LGBTQIAP+ na vida acadêmica, $43,40 \%$ dos entrevistados relataram ser pouco abordado; $35,84 \%$ afirmaram não ser abordado; $18,87 \%$ moderadamente abordado e apenas 1,89\% refere ter sido totalmente abordado na Tabela 1. 
Para tanto, ao serem questionados os estudantes de Enfermagem relataram que a sexualidade vem provocando um debate relacionado à descriminação e exclusão da população LGBTQIAP+, entre os profissionais (biomédicos, políticos, cientistas sociais e antropológicos) há essa discussão, que inclui também a saúde e retrata o cuidado e o acesso aos serviços de saúde, afinal os estudos mostram que a população LGBTQIAP+ relata problemas ao se consultar com os profissionais de saúde, pois sente receio em informar a sua identidade de gênero ou orientação sexual .

Foi possível observar que houve maior proporção de discentes que se sentiam capacitados em realizar atendimento entre aqueles que conheciam plenamente o significado da sigla LGBTQIAP+ $(p=0,004)$, saber diferenciar sexo biológico, identidade de gênero, expressão de gênero e orientação sexual $(p=0,015)$, reconhece a diferença entre transexual, homossexual e transgêneros $(0,116)$, estar ciente da existência de alguma política de amparo à saúde para essa população $(p=0,005)$ e percepção de peculiaridades do público LGBTQIAP+ que necessitam de maior atenção no âmbito da saúde $(p=0,030)$ Tabela 2.

Tabela 2 - Conhecimento dos discentes de acordo com a capacidade em realizar o atendimento à população LGBTQIAP+ e contato dos discentes com a temática durante a trajetória acadêmica.

\begin{tabular}{|c|c|c|c|c|c|c|c|}
\hline & & \multicolumn{2}{|c|}{$\begin{array}{c}\text { Sente-se capacitado em atender } \\
\text { um indivíduo LGBTQIAP+e } \\
\text { suas particularidades }\end{array}$} & & \multicolumn{2}{|c|}{$\begin{array}{c}\text { Abordagem em alguma disciplina } \\
\text { sobre a melhor conduta frente a } \\
\text { comunidade LGBTQIAP+ }\end{array}$} & \\
\hline & & Não/ Pouco & $\begin{array}{l}\text { Moderado/ } \\
\text { Totalmente }\end{array}$ & & Não/ Pouco & $\begin{array}{l}\text { Moderado/ } \\
\text { Totalmente }\end{array}$ & \\
\hline & $\mathbf{N}(\%)$ & $\mathbf{N}(\%)$ & $\mathbf{N}(\%)$ & $\mathbf{p}$ & $\mathbf{N}(\%)$ & $\mathbf{N}(\%)$ & $\mathbf{p}$ \\
\hline $\begin{array}{l}\text { Conhece o significado } \\
\text { da sigla LGBTQIAP+ }\end{array}$ & & & & 0,004 & & & 0,004 \\
\hline Não/ Parcialmente & $25(47,17)$ & $15(28,30)$ & $10(18,87)$ & & $24(47,17)$ & $1(1,98)$ & \\
\hline Sim & $28(52,83)$ & $6(11,32)$ & $22(41,51)$ & & $18(33,96)$ & $10(18,87)$ & \\
\hline $\begin{array}{l}\text { Sabe diferenciar sexo } \\
\text { biológico, identidade } \\
\text { de gênero, expressão } \\
\text { de gênero e } \\
\text { orientação sexual }\end{array}$ & & & & 0,465 & & & 0,015 \\
\hline Não/Parcialmente & $27(50,94)$ & $12(22,64)$ & $15(28,30)$ & & $25(47,17)$ & $2(3,77)$ & \\
\hline Sim & $26(49,06)$ & $9(16,98)$ & $17(32,08)$ & & $17(32,08)$ & $9(16,98)$ & \\
\hline $\begin{array}{l}\text { Reconhece a } \\
\text { diferença entre } \\
\text { transexual, } \\
\text { homossexual e } \\
\text { transgênero }\end{array}$ & & & & 0,191 & & & 0,116 \\
\hline Não/Não sabe informar & $8(15,09)$ & $5(9,43)$ & $3(5,66)$ & & $8(15,09)$ & - & \\
\hline Sim & $45(84,91)$ & $17(32,08)$ & $28(52,83)$ & & $34(64,15)$ & $11(20,75)$ & \\
\hline $\begin{array}{l}\text { Ciente de alguma } \\
\text { política de amparo à } \\
\text { saúde para essa } \\
\text { população }\end{array}$ & & & & 0,042 & & & 0,005 \\
\hline Não/Não sabe informar & $44(83,02)$ & $21(39,62)$ & $23(43,40)$ & & $38(71,70)$ & $6(11,32)$ & \\
\hline Sim & $9(16,98)$ & $1(1,89)$ & $8(15,09)$ & & $4(7,55)$ & $5(9,43)$ & \\
\hline $\begin{array}{l}\text { Percepção de } \\
\text { peculiaridades do } \\
\text { público } \\
\text { LGBTQIAP+ que } \\
\text { necessitam de maior } \\
\text { atenção no âmbito da } \\
\text { saúde }\end{array}$ & & & & 0,632 & & & 0,03 \\
\hline Não/Não sabe informar & $15(28,30)$ & $7(13,21)$ & $8(15,09)$ & & $9(16,98)$ & $6(11,32)$ & \\
\hline Sim & $38(71,70)$ & $15(28,30)$ & $23(43,40)$ & & $33(62,26)$ & $5(9,43)$ & \\
\hline
\end{tabular}

Fonte: Gomes TMC, et al., 2020. 
De acordo com o Gráfico 1 abaixo a maioria dos entrevistados, 28\%, classificam-se com pouca aptidão para um atendimento de forma satisfatória e respeitosa ao público LGBTQIAP+, seguidos de $13 \%$ com percepção moderadamente apto, $9 \%$ não se identificam aptos e $3 \%$ consideram-se aptos. Portanto, verificou-se um baixo índice de capacitação dos discentes para o atendimento ao público LGBTQIAP+, segundo a pesquisa foi constatado que a maior parte dos estudantes de enfermagem avaliam-se com pouca aptidão para realização de um atendimento ao público em questão.

Gráfico 1 - Nível de aptidão dos acadêmicos de enfermagem para atendimento ao público LGBTQIAP+.

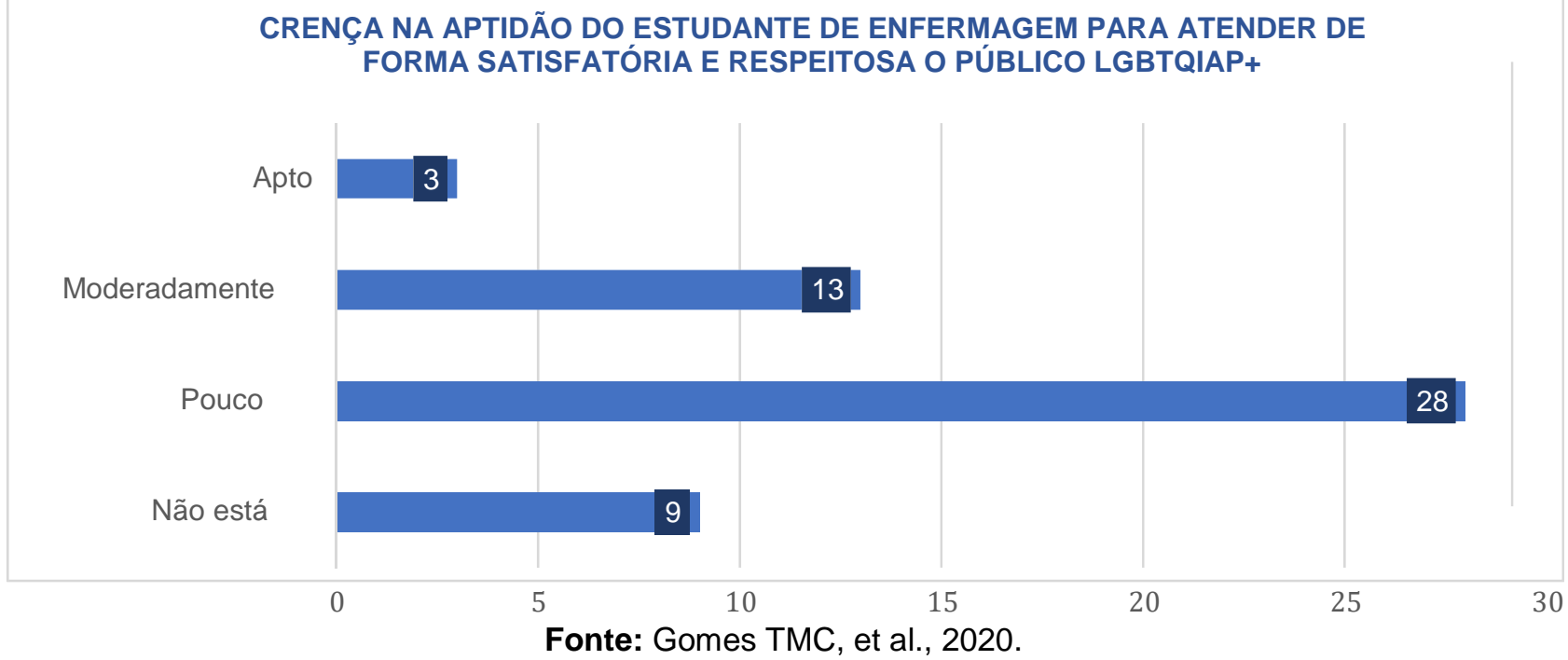

Ao realizar a análise do Gráfico 2, identificou-se um índice de 19\% dos estudantes que acreditam que o profissional de saúde deve ter uma base de conhecimento para promover atendimento humanizado que abranja todas as particularidades, sendo que $13 \%$ acreditam que há falhas, embora existam protocolos de cuidados para população, já $10 \%$ dos discentes não sabem informar. Além disso, $6 \%$ acreditam que não existe assistência adequada ao público e $5 \%$ acreditam que se deve proporcionar atendimento igualitário.

Gráfico 2 - Percepção sobre as questões de gênero e as responsabilidades dos serviços de saúde.

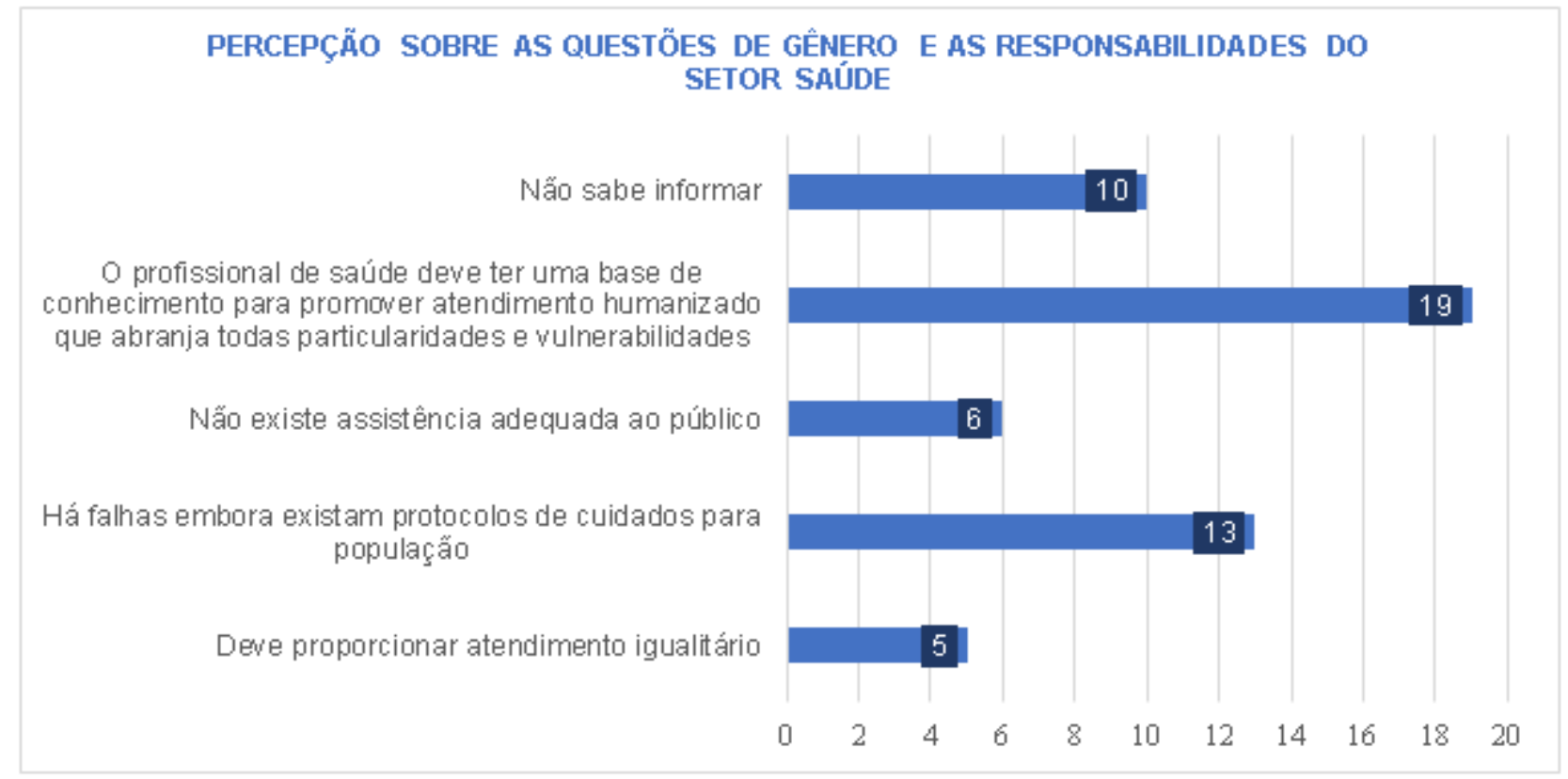

Fonte: Gomes TMC, et al., 2020. 
Diante do Gráfico 3 em questão, nota-se que dois resultados são apresentados. Primeiramente, em relação à abordagem da temática na instituição de ensino, os parâmetros indicam que $42 \%$ dos acadêmicos acreditam que deve haver mudanças na forma de atendimento atual ao paciente LGBTQIAP+, sendo que $6 \%$ não sabem informar e $5 \%$ acreditam que não deve haver mudanças na forma de atendimento a população. Por outro lado, em relação ao segundo resultado apresentado, $45 \%$ dos acadêmicos acreditam que deve haver mudanças na abordagem do assunto pela sua instituição de ensino, por outro lado, $6 \%$ acreditam que não deve haver mudanças e $2 \%$ não sabem informar.

Grafico 3 - Abordagem da temática e ensino sobre o atendimento à população LGBTQIAP+.

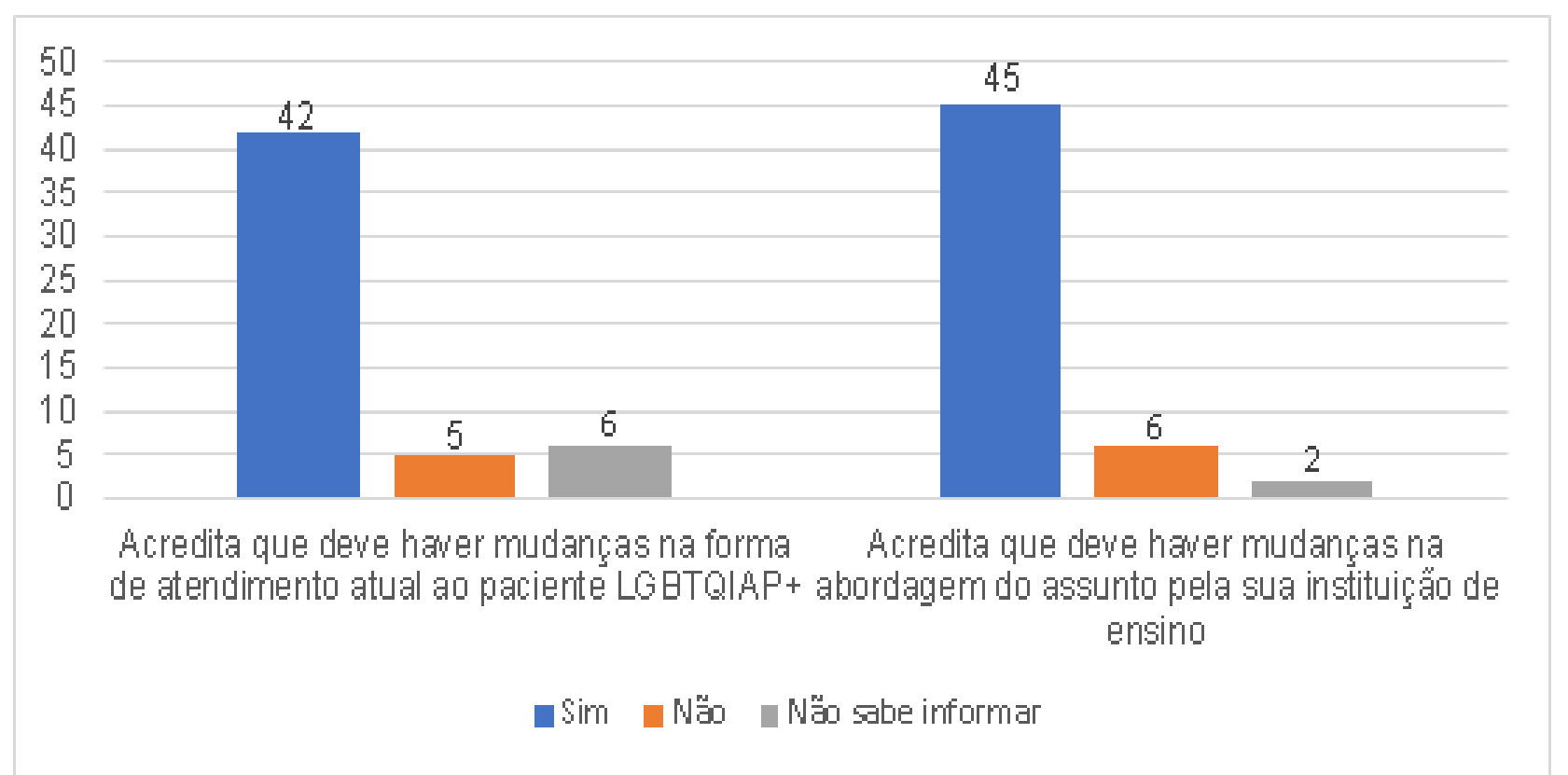

Fonte: Gomes TMC, et al., 2020.

\section{DISCUSSÃO}

A maioria dos discentes do curso de enfermagem é do sexo feminino. As mulheres conseguiram reverter um quadro e consolidar uma nova realidade, em que são mais escolarizadas do que os homens, "tradicionalmente" do sexo masculino. O recorte da população de nível universitário reflete o quanto a mudança foi rápida e marcante. Assim, a entrada feminina na universidade revela diferenciações históricas fundamentais nos tipos de inserção social.

Para Pereira SOGP e Nunes JB (2018), no que concerne à desigualdade de gênero, a dificuldade de acesso e permanência no ensino superior é complexa, embora seja reconhecida a expansão do acesso e a significativa presença do público feminino nas universidades. Essa constatação não exclui, porém, as desigualdades existentes nesse espaço no que se refere ao gênero, raça/etnia e condição social.

Conforme Silva ALR, et al. (2019) afirma que há uma notável relação entre os hábitos culturais do gênero feminino e a área de enfermagem. Ou seja, o papel de cuidar está diretamente associado às mulheres, uma vez que, numa sociedade patriarcal, convencionou-se que o público feminino tem uma maior obrigação com o cuidar das pessoas, por isso a enfermagem é historicamente correlacionada ao gênero feminino.

Foi possivel observer neste estudo que a faixa etária ou até mesmo o estado civil influenciam as expectativas do indivíduo em desenvolver suas habilidades no decorrer do período. Os resultados mostraram que os alunos do $9^{\circ}$ e $10^{\circ}$ período têm uma compreensão melhor do assunto, pois apresentam uma visão mais prudente e capacitada para o atendimento, especificamente para os grupos de gêneros. A prática está ainda pouco desenvolvida e priorizada, na qual esses estudantes e futuros profissionais recebem pouca qualificação. 
Segundo Perreira EO, et al. (2017) entendem a sexualidade e o gênero não como categorias fixas, mas em constante movimento e não classificáveis, permitindo construir uma ideia de como esses temas vêm sendo tratados no cenário social contemporâneo como algo fronteiriço, quer dizer, que contorna as fronteiras morais daquilo que as sociedades consideram como algo natural e normal.

Com isso, os resultados mostram que os estudantes que obtêm conhecimento do significado da sigla LGBTQIAP+ estão mais preparados para atender esse grupo. É conveniente destacar que as políticas de saúde voltadas para essa população foram construídas e estimuladas de forma compartilhada com os movimentos sociais.

Esse fato trouxe empoderamento a tal questão, já que, mesmo com todos os avanços advindos na área da saúde, o preconceito nos estabelecimentos de saúde e a invisibilidade entre os profissionais ocultaram ainda mais essa população, fazendo dos espaços lugares incapazes para promover saúde e atender às reais necessidades da comunidade LGBTQIAP+ (NEGREIROS FRN, et al., 2019).

Considerando que esse público historicamente foi negligenciado, sofreu e ainda sofre preconceitos, e que atualmente necessita de um atendimento que visa à equidade para um acesso de qualidade na assistência de saúde prestada na Atenção Básica, surge à necessidade de conhecer e avaliar o papel do profissional enfermeiro na implementação da política LGBT (GUIMARÃES NP, et al., 2020).

Já para Santana ADS, et al. (2020) evidenciam em seu estudo que, mesmo sendo lei que a saúde é um direito de todos e posteriormente ter sido implantada a Política Nacional de Saúde Integral à Lésbicas, Gays, Bissexuais, Travestis e Transexuais (LGBTQIAP+), o acesso à saúde para a respectiva população ainda sofre grandes obstáculos.

O estudo explanado constatou que a maioria dos estudantes tem como entendimento que os acadêmicos de saúde devem acolher e ter conhecimento sobre todas as especificidades, pois quando o público é exposto a qualquer tipo de preconceito ou discriminação por parte de profissionais de saúde em serviço, o sistema de saúde tende a ter pouca eficiência. Desta forma, os serviços de atendimento devem ser ofertados independente do nível de dificuldade, sem empecilhos ou obstáculos, tendo consideração e prioridade aos que mais necessitam, oferecendo uma atenção especial às finalidades e necessidades na saúde da população LGBTQIAP+.

Diante disso, Silva ACA, et al. (2020) retratam obstáculos que a população LGBTQIAP+ se depara diante do acesso ao serviço de saúde, como os preconceitos, a desigualdade e até distinções. Vista disso, os serviços nas instituições devem assegurar uma atenção com competência e reverência, solucionando seus objetivos de maneira íntegra e de qualidade.

Os resultados mostram que a desigualdade e as distinções são consideradas uma razão que determina um processo de tormento e enfermidade. De acordo com Albuquerque GA, et al. (2016b) existe preconceito voltado a essa população pelos profissionais de saúde. Sendo assim, esse público tem receio ao expressar sua sexualidade, pois temem ter rejeição por parte dos profissionais e na condição de atendimento prestado.

Segundo Lovison R, et al. (2019), referente às práticas dos profissionais, entende-se que a integralidade da atenção passa por um processo de trabalho em saúde que se interessa pela produção do cuidado eficaz, humanizado e centrado nas necessidades dos usuários, tanto individual quanto coletivamente e deve partir da organização dos processos de trabalho em qualquer nível do sistema, inclusive na atenção básica, por meio de diretrizes como o acolhimento, o vínculo e o conceito ampliado de saúde, tendo as necessidades do usuário como centro do pensamento e da produção do cuidado da equipe.

Foi possivel observar neste estudo que a grande parte dos discentes entrevistados acredita-se que devem ocorrer mudanças no atendimento oferecido à população LGBTQIAP+, quanto na abordagem dessa temática nas instituições, entretanto nem sempre as instituições se mostram capazes de enfrentar as diferenças, em particular com as questões relacionadas à sexualidade. Esse conflito traz sérios efeitos para todos os discentes, visto que atrapalha seus conhecimentos, o processo de aprendizagem, e dificulta a contribuição para o seu desenvolvimento. 
É importante reconhecer que os processos de diminuir o preconceito contra a população LGBTQIAP+ acarretam agravos à saúde, pois a politica tem como propósito desenvolver amplas recomendações aos diferentes setores do governo objetivando assegurar ações quecombatessem os diversos fatores de vulnerabilidade aos quais a população LGBTQIAP+ está exposta, sobretudo, a promoção da equidade e do acesso a ações de saúde prioritárias a esse público, com foco na realização efetiva da estruturação de uma política nacional de saúde, com produção de conhecimentos e com capacitações dos profissionais de saúde para o adequado atendimento (ZANE LF e TERRA MF, 2019).

Diante dos resultados obtidos no estudo, foi a respeito que tinha pouco atributo para um atendimento com todo zelo e atenção necessária direcionada para a população, e ressaltam a falta de abordagem dessa temática diante das atividades acadêmicas. Segundo Nietshe EA, et al. (2018) em seu estudo, os acadêmicos manifestaram um conflito ao expor seus entendimentos quanto à temática.

O que foi observado neste estudo é que os discentes de enfermagem durante o atendimento não mostraram compreensão e nem prática para a atenção voltada a esse público, destaca-se a importância de atividades construtivas a fim de proporcionar um aprendizado e experiência para os alunos durante a formação acadêmica, de modo que sejam incentivados a conduzir as ações, objetivando o desenvolvimento de habilidades.

Um dos dados importante, diante do resultado obtido no estudo, foi que a ausência ou pobreza de aprendizado gera empecilhos no atendimento prestado. Assim, infere-se que existe escassez do conhecimento dos estudantes referente a essa temática, refletindo a pouca nitidez e pouco reconhecimento das especificidades de atenção ao público LGBTQIAP+.

Com isso, a atribuição do enfermeiro consiste na responsabilidade das ações desenvolvidas para o alcance da excelência na assistência de saúde à população LGBTQIAP+, desde o jovem adolescente até o idoso deste público-alvo, baseando-se nas orientações referentes às Infecções Sexualmente Transmissíveis (IST'S), prevenindo contra possíveis patologias advindas delas.

Portanto, como principal atuação do enfermeiro a essa população, está a garantia de uma assistência em saúde que acolha com dignidade e de forma humanizada, já iniciada na atenção primária de saúde até a atenção terciária de saúde, respeitando as diferenças mesmo não as aceitando, mas que seja fundamental para a promoção de saúde integral e de equidade para esse público.

\section{CONCLUSÃO}

Foi possiviel concluir que, o presente estudo permitiu identificar o conhecimento dos acadêmicos do curso de Enfermagem do Centro Universitário Estácio de Sergipe em relação ao atendimento de saúde para a população LGBTQIAP+ e cerca dos paradigmas da diversidade sexual, na qual, evidenciou que o nível de conhecimento dos estudantes acerca das singularidades e políticas de saúde direcionadas à está população é determinante para uma oferta direcionada à uma assistência integral que reconheça os contextos de vulnerabilidade e exclusão a que está exposta. Com isso, tendo em vista os argumentos apresentados, têm a percepção que para propiciar acolhimento humanizado e de qualidade, deve-se ter o conhecimento sobre o tema e sobre as políticas públicas já existentes.

\section{REFERÊNCIAS}

1. ALBUQUERQUE GA, et al. Homossexualidade e o direito à saúde: um desafio para as políticas públicas de saúde no Brasil. Saúde debate, 2016a; 37(98):516-524.

2. ALBUQUERQUE GA, et al. Violência psicológica em lésbicas, gays, bissexuais, travestis e transexuais no interior do Ceará, Brasil. Revista Saúde Debate, 2016b;40(109):100-111.

3. ALMEIDA JSM, et al. Cuidar de pessoas transexuais na ótica dos residentes de enfermagem [Care for transgender people from the nursing resident's perspective] [Cuidar de personas transexuales en la óptica de los residentes de enfermería]. Revista Enfermagem UERJ, 2018; 26: 32030.

4. BARDIN L. Análise de conteúdo. 70nd ed. São Paulo, 2011;229p.

5. CARDOSO MR, LUZ NS. Saúde e População LGBT: Demandas e Especificidades em Questão. Rev. Psicologia: Ciência E Profissão, 2017; 32(3):552-563. 
6. FERREIRA BO, et al. Diversidade de gênero e acesso ao Sistema Único de Saúde. Revista Brasileira Promoção Saúde, 2018; 31(1):1-10.

7. FIGUEROA MN, et al. A formação relacionada com a sexualidade humana na percepção de estudantes de enfermagem. Revista de Enfermagem Referência IVạ Série. 2017; 4(15)123-158.

8. GOMES TMC, et al. O movimento social pelo reconhecimento da cidadania LGBT, Revista de Educação Ciência e Tecnologia. 2020;8(1)231-245.

9. GUIMARÕES NP, et al. Avaliação da implementação da Política Nacional de Saúde Integral à população LGBT em um município da região Sudeste do Brasil. Reciis - Revista Eletronica Comunicação Informação Inovação de Saúde, 2020; 14(2):372-85.

10. LOPES LP, et al. Diversidades de gêneros e acesso à saúde: concepção dos estudantes de medicina e enfermagem do centro universitário de Patos de Minas. Brazilian Journal of Health Review. 2019;2(4)32863302.

11. LOVISON R, et al. Travestis e transexuais: despindo as percepções acerca do acesso e assistência em saúde. Enfermagem em Foco, 2019;10(5):167-172.

12. NEGREIROS FRN, et al. Saúde de Lésbicas, Gays, Bissexuais, Travestis e Transexuais: da Formação Médica à Atuação Profissional. Revista Brasileira de Educação Médica, 2019;43(1):23-31.

13. NIETSCHE EA, et al. Formação do enfermeiro para o cuidado à população homossexual e bissexual: percepção do discente. Revista Baiana de Enfermagem, 2018;32(2): 51-74.

14. PEREIRA EO, et al. Unidades básicas de saúde em Teresina-PI e o acesso da população LGBT: O que pensam médico? Revista Tempus, actas de saúde colet, 2017;11(1):51-67.

15. PEREIRA SOGP, NUNES JB. A Política Nacional de Saúde Integral de Lésbicas, Gays, Bissexuais e Transgêneros (LGBT) e o acesso ao Processo Transexualizador no Sistema Único de Saúde (SUS): avanços e desafios. Revista Ciência \& Saúde Coletiva, 2018; 22(5):1509-1520.

16. SANTANA ADS. Dificuldades no acesso aos serviços de Saúde por Lésbicas, Gays, Bissexuais e Transgêneros. Revista de enfermagem UFPE, 2020;14:243-211.

17. SANTOS JS, et al. Saúde da população LGBTI+ na Atenção Primária à Saúde e a inserção da Enfermagem. Revista Escola Anna Nery, 2019;23(4):21-30.

18. SILVA ACA, et al. Implementação da Política Nacional de Saúde Integral de Lésbicas, Gays, Bissexuais, Travestis e Transexuais (PNSI LGBT) no Paraná, Brasil. Interface (Botucatu), 2020;24(1):190-568.

19. SILVA ALR, et al. Representações sociais de trabalhadores da atenção básica à saúde sobre pessoas LGBT. Trabalho, educação e saúde, 2019;17(2): 23-35.

20. ZANI LF, TERRA MF. Conhecimentos sobre identidade de gênero e orientação sexual entre graduandos/as de enfermagem. Journal Health NPEPS, 2019; 4(2):167-179. 